\title{
Scene Text Detection Using YOLOv4 Framework
}

\author{
Nisarg Gandhewar', S. R. Tandan ${ }^{2}$ and Rohit Miri ${ }^{3}$ \\ ${ }^{1} P h D$ Scholar, CSE Dept, CVRU, Kota, (Chhattisgarh), India \\ ${ }^{2,3}$ Associate Professor, CSE Dept, CVRU, Kota, (Chhattisgarh), India
}

\section{ABSTRACT}

Extraction of printed information from different certifiable pictures in machine readable format is treated as tricky chore in the domain of computer vision. This has gained plenty of consideration due to its extensive applications in industries and our daily life. Recently developed deep learning based techniques for detection of text have obtained encouraging results with respect to different standard datasets. But, they typically fall behind when exposed to tricky situation. Still there is a contest between speed and accuracy for text detection. Traditionally for text detection mostly the two stage detector techniques like Faster-RCNN, Fast-RCNN and R-CNN were explored. In this work, we put forward a straightforward pipeline for detecting text in common scene pictures. Here we used recent state of art one stage object detection framework known as YOLOv4 (You Only Look Once version 4) with darknet framework which is comparatively faster and accurate, contrast to existing object detectors. It gives us speedy and precise detection of text from common scene pictures. Experiments performed on state of art datasets including ICDAR 2015, ICDAR 2013, ICDAR 2003, SVT, IIIT5K, MSRA-TD500 display that the proposed pipeline significantly performs better except for ICDAR2015, compare to existing techniques with respect to correctness.

\section{KEY WORDS: COMPUTER VISION, OBJECT DETECTION, DARKNET, TEXT DETECTION.}

\section{INTRODUCTION}

Text is the key discoveries of humanity, which has played a vital role in human's life since from earliest times. The specific information embodied in the image is very useful in a broad range of applications because of its realistic applications in updating inventory, understanding scene, analyzing documents, navigation of robot, industrial automation and retrieval of image. Text is a major aspect in a variety of scenes and acts as a one of the primary way for people to communicate among each other. Recently detection and recognition of text from an image have turn

\section{ARTICLE INFORMATION}

*Corresponding Author: nisarg.gandhewar@gmail.com Received 18th Oct 2020 Accepted after revision 29th Dec 2020 Print ISSN: 0974-6455 Online ISSN: 2321-4007 CODEN: BBRCBA

Thomson Reuters ISI Web of Science Clarivate Analytics USA and Crossref Indexed Journal

\section{Clarivate
Analytics}

NAAS Journal Score 2020 (4.31)

A Society of Science and Nature Publication,

Bhopal India 2020. All rights reserved.

Online Contents Available at: http//www.bbrc.in/

Doi: http://dx.doi.org/10.21786/bbrc/13.14/43 out to be a trending topic of research in several fields like document analysis and computer vision. Object detection is treated as more complex problem than classification, which can also recognize objects but doesn't let you know precisely where the object is positioned in the image and it didn't work for images that includes more than one object. The chore of detecting object is to uncover all concerned objects in the image, and find out their category with exact position. It's one of the main issues in the computer vision field. A range of objects has dissimilar appearances, postures and shapes, coupled with the interference of occlusion, lighting and other factors during imaging, target detection has at all times been the most tricky problem in the computer vision field. Object detection job has shown promising result with increasing use of techniques based on deep learning framework. Current techniques consider text as precise entity and explore general object detection framework to solve text localization problem. 
As compared to conventionally used OCR system, detection and recognition of scene text can adapt to any scene, exclusive of sole needs, like traffic signs, hoardings and different bills. Recently, deep learning based object detection techniques have made great impact in the community of computer vision to solve many problems, which can be generally grouped into two types. One is object localization dependent on the area of intrigue. Here the primary guideline is to utilize locale suggestion to produce post-chose targets, and afterward use Convolutional Neural Network to deal with them. They ordinarily have a high precision, however are frequently insufficient progressively. It incorporates different techniques of RCNN family. Another type of object detection techniques are depend on regression technique, that accepts an issue of detection as a problem of regression to resolve and straightforwardly predicts target object position and its class in one stage.

These types of techniques usually have faster speed but low accuracy and it includes YOLO, SSD, YOLOv2, YOLOv3. As compared to the traditional family of RCNN, YOLO has better speculation when used in different fields. Yet object detection begins growing in the last few years, the contest left over fierce. YOLOv4 not only achieve very good accuracy but also have high processing frame rate. It attains an accuracy of 43.5\% AP and 65.7\% A Pover the MS COCO dataset along with an around 65 FPS inference speed over Tesla V100. In object detection, high accuracy is not the only considerable factor but we want the model to run smoothly in the edge devices. YOLOv4 obtains promising outcome at a continuous speed over the MS-COCO dataset. Advanced precise models require numerous GPUs for training with an enormous mini batch size, and doing this with one GPU makes the preparation truly moderate and illogical. YOLOv4 tends to this worry by making an object locator that can be prepared on a sole GPU with a smaller mini batch size [A. Bochovsky, 2020]. So as to accomplish effective scene text detector, this paper chooses a pipeline dependent on YOLOv4.

Related Work: Detection of text from common scene pictures involves locating text in pictures and creates bounding boxes for it. Traditionally connected component based, Sliding window based, Texture based techniques have been extensively exploit for locating text but in recent year's deep learning-based techniques have witness incredible enhancement for detection of text. Now a day's text detection techniques are characterized into two classes as general object detection and technique based on segmentation [H Lin, 2019].

General Object Detection Based Techniques: These types of techniques generally anticipate candidate bounding boxes legitimately by considering objects as text. This type of techniques is frequently skilled by using annotations based on bounding box. Techniques based on General object detection are classified into two classes as one stage and two stage detector technique. Even though the two stage detector achieves top accuracy, recent works dependent on one stage detector additionally uncover quicker text detection with equivalent exactness. Dissimilar to FCN based techniques, this class of techniques employs text as a particular object and gives various powerful frameworks of object detection like SSD, Retina Net, YOLO, RCNN, Faster RCNN and Fast RCNN to detect text or words from images directly.

Linjie Deng et al. proposed a technique rely upon RetinaNet for arbitrary oriented detection of text, having aim to incorporate the learning mechanism borrowed from two stage RCNN structure into the one stage detector [L. Deng , 2019]. Minghui Liao et al. present text detection technique TextBoxes which is fit for identifying text in a sole network, having no post process excluding non max suppression [M. Liao, 2017]. Minghui Liao et al. also developed TextBoxes++ approach based on SSD for multi oriented detection of scene text having both high precision and proficiency [M. Liao,2018]. Zhida Huang et al. developed approach based on Mask RCNN having backbone of (PAN) Pyramid Attention Network meant for multi oriented detection of text [Z. Huang, 2019]. Pranav Adarsh et al. proposed YOLO v3-Tiny one stage improved model based on YOLO speeds up object detection while guarantees the precision of the outcome [P. Adarsh, 2020].

Segmentation Based Techniques: These types of techniques are inspired by FCN, generally use to deliver more explicit text regions. Those techniques are experimented over the multi size text detection, yet they are ineffectual while locating singular text lines and words. These techniques first obtain blocks of text from the division map produced by FCN and then by complex post processing the bounding boxes of text are generated. Dan Deng proposed PixelLink an instance segmentation based technique where text sample is first segregated by connecting pixels inside the identical instance collectively. Bounding box for text is then obtained from the segmentation output directly, exclusive of location regression [D. Deng, 2018]. Xue et al. use text fringe semantics for exact confinement of texts in the scene [C. Xue, 2018]. Wang et al. presents PSENet to locate text sample of random shapes and produce the diverse size of kernels for every sample of text and steadily enlarge the minimal scale kernel to the sample of text of entire shape [W. Wang, 2019]. Yao et al. consider identification of text as a semantic segmentation issue and identify curved texts in diverse scene images [M. Liao, 2020].

\section{METHODOLOGY}

The proposed pipeline for detection of text is demonstrated in fig.1 Here first an python based image annotation tool called Labelimg is used to annotate all images and prepare the dataset like ICDAR 2015, ICDAR 2013, ICDAR 2003, SVT, IIIT5K, MSRA-TD500 in a format accepted by YOLO framework.

The Labelimg generates the text file for every image containing information like object class, $\mathrm{x}$ centre, $\mathrm{y}$ centre, height and width, all these value's were normalized between 0 and 1 . These prepared datasets are submitted 
individually to YOLOv4 framework for training which makes use of a novel backbone CSPDarknet53 that can improve the learning capability of CNN. So as to enlarge the receptive field and split out the most significant context features, the spatial pyramid pooling block is added over CSPDarknet53. The path aggregation network (PANet) is used as neck for parameter aggregation for different detector levels. The use of the neck block is to add extra layers between the backbone and the head. Finally the head block is used to locate bounding boxes and classify what's inside each box similar to YOLOV3 with dense prediction.

Figure 1: Proposed Text Detection Pipeline

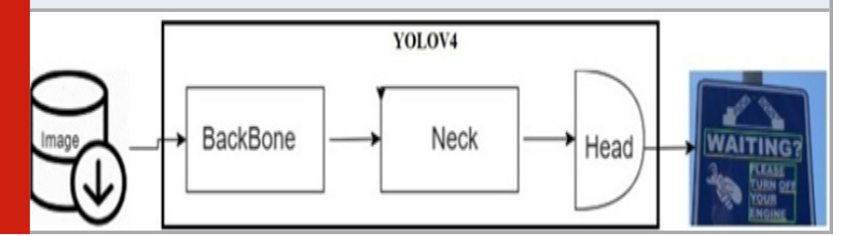

In order to train a model over the different benchmark dataset, we put the values of hyper parameters as, batch $=64$, subdivisions $=32$, width $=608$, height $=608$, channel $=3$, momentum $=0.949$, decay $=0.0005$, saturation $=1.5$, exposure $=1.5$, hue $=0.1$, learning rate $=0.00261$, maximum batches $=4000$, Policy $=$ steps and steps $=80 \%$ and $90 \%$ of maximum batches as 3200,3600 and filters $=18$. Here we use only first 137 layers out of 162 from yolov4.weight which is trained on MS COCO dataset to get better result.

Table 1. Outcome of text detection technique on IIIT5K dataset

\begin{tabular}{|l|c|c|c|}
\hline Technique & P & R & F \\
\hline Our YOLOv4 & 97 & 97 & 97 \\
\hline
\end{tabular}

Table 2. Outcome of text detection techniques on SVT dataset

\begin{tabular}{|l|c|c|c|}
\hline Technique & P & R & F \\
\hline Our YOLOv4 & 87 & 67 & 76 \\
\hline Tian [X. Liu, 2019] & 68 & 65 & 66 \\
\hline Zhang [Z. Zhang, 2015] & 68 & 53 & 60 \\
\hline Rong [X Rong, 2017] & 29 & 27 & 28 \\
\hline Gupta [X. Liu, 2019] & 26.20 & 27.4 & 26.7 \\
\hline Jaderberg [H. Lin, 2019] & 53.6 & 62.8 & 46.8 \\
\hline Kittler [H Lin, 2019] & 55 & 81 & 62 \\
\hline Kasar [T. Kasar, 2007] & 70 & 71 & 69 \\
\hline
\end{tabular}

\section{RESULTS AND DISCUSSION}

The experiment is carried out on system with Tesla T4 GPU, with 12GB of VRAM, using open source framework called as Darknet. So as to estimate the feat of proposed pipeline and different existing techniques, parameters like F-measure- $(\mathrm{F})$, precision- $(\mathrm{P})$ and Recall- $(\mathrm{R})$ are considered. An experiment is carried on state of art dataset like ICDAR 2015, ICDAR 2013, ICDAR 2003, SVT, IIIT5K, MSRA-TD500 and result obtained by our pipeline and existing techniques are summarized in below tables.

Table 3. Outcome of text detection techniques on MSRATD 500 dataset

\begin{tabular}{|l|c|c|c|}
\hline Technique & $\mathrm{P}$ & $\mathrm{R}$ & $\mathrm{F}$ \\
\hline Our YOLOv4 & 83 & 78 & 81 \\
\hline Zhou [X. Zhou, 2017] & 87.2 & 67.4 & 76.08 \\
\hline Zhang [Z. Zhang, 2015] & 83 & 67 & 74 \\
\hline He [X. Liu, 2016] & 77 & 70 & 74 \\
\hline Turki[X Liu,2016] & 72 & 79 & 75.3 \\
\hline Liao [M. Liao, 2017] & 87 & 73 & 79 \\
\hline Deng[H. Lin,2019] & 83 & 73.2 & 77.8 \\
\hline Liu [H. Lin, 2019] & 84.5 & 77.1 & 80.6 \\
\hline
\end{tabular}

Table 4. Outcome of text detection techniques on ICDAR 2013 dataset

\begin{tabular}{|l|c|c|c|}
\hline Technique & $P$ & $R$ & $F$ \\
\hline Our YOLOv4 & 89 & 94 & 91 \\
\hline Zhong [X Liu,2019] & 93 & 86.7 & 89.7 \\
\hline He [X Liu,2019] & 92 & 81 & 86 \\
\hline Gupta [H Lin,2019] & 92 & 75.5 & 83 \\
\hline Liao[M Liao,2017] & 89 & 83 & 86 \\
\hline Zhang [Z. Zhang, 2015] & 88 & 74 & 80 \\
\hline Lyu [H Lin,2019] & 92 & 84.4 & 88 \\
\hline
\end{tabular}

Table 5. Outcome of text detection techniques on ICDAR 2015 dataset

\begin{tabular}{|l|c|c|c|}
\hline Technique & $\mathrm{P}$ & $\mathrm{R}$ & $\mathrm{F}$ \\
\hline Our YOLOv4 & 69 & 68 & 69 \\
\hline Zhou [X. Zhou, 2017] & 83.27 & 78.3 & 80.7 \\
\hline He [X Liu,2019] & 82 & 80 & 81 \\
\hline Liao [M Liao,2017] & 87.8 & 78.5 & 82.9 \\
\hline Lyu [H Lin,2019] & 89.5 & 79.7 & 84.3 \\
\hline Zhong [X Liu,2019] & 89 & 83 & 86 \\
\hline Xie [W Wang, 2019] & 84 & 81.9 & 82.9 \\
\hline Wang [H Lin,2019] & 86.9 & 84.5 & 85.7 \\
\hline
\end{tabular}

For IIIT5K dataset our pipeline got impressive result shown in table1, whereas for SVT dataset our pipeline got better result for precision and F-measure compare to existing techniques whereas Kittler got better result for recall shown in table 2, similarly for MSRA-TD 500 dataset our pipeline got better result for F-measure compare to existing techniques whereas Turkey got better result for recall and Zhou for precision shown in table3, 
also for ICDAR 2013 dataset our pipeline got better result for Recall and F-measure compare to existing techniques whereas Zhong got better result for precision shown in table 4. For ICDAR 2015 dataset Zhong et al. got better result for F-measure while Lyu et al. got better result for precision and Wang et al. for recall as shown in table5. For ICDAR 2003 dataset our pipeline got better result for precision and F-measure while Kittler got better result for recall as shown in table 6 .

Table 6. Outcome of text detection techniques on ICDAR 2003 dataset

\begin{tabular}{|l|c|c|c|}
\hline Technique & P & R & F \\
\hline Our YOLOv4 & 83 & 82 & 83 \\
\hline Kittler [H Lin,2019] & 75 & 89 & 78 \\
\hline Kasar [T. Kasar, 2007] & 72 & 64 & 65 \\
\hline Sauvola [X Liu, 2019] & 65 & 83 & 67 \\
\hline Howe [N.Howe,2011] & 76 & 84 & 76 \\
\hline
\end{tabular}

The values shown in bold in all tables represent the best result for respective performance metrics. Overall our pipeline got better results over the state of art datasets like ICDAR 2013, ICDAR 2003, SVT, IIIT5K, MSRA-TD500 except for ICDAR 2015 as compare to existing techniques used for detection of text.

\section{CONCLUSION}

There exist several techniques with a compromise in speed performance and exactness of outcome to identify text in scene images. In this manuscript, we have presented a simple pipeline for locating text using current state of art object detection YOLOv4 framework and the working principle with training process of YOLOv4 are illustrated in detail. The efficacy of our proposed pipeline has been verified on several public benchmarks datasets like ICDAR 2015, ICDAR 2013, ICDAR 2003, SVT, IIIT5K, MSRA-TD-500 by considering commonly used performance metrics like precision, recall and F-measure. Our pipeline shows promising result as compare to existing techniques of text detection over several benchmark datasets except ICDAR 2015.

\section{REFERENCES}

A. Bochovsky, C. Wang, H. Yuan, "YOLOv4- Optimal Speed and Accuracy of Object-Detection”, Comp Vis and Patt Recogn. arXiv:2004.10934, submitted on 23 Apr 2020, to Cornell University.
C. Xue, S. Lu, F. Zhan," Accurate Scene-TextDetection through Border Semantics Awareness and Bootstrapping”, ECCV, 2018.

D. Deng, H. Liu," Pixel-Link- Detecting Scene-Text via Instance Segmentation”, AAAI, 2018.

H. Lin, P. Yang, "Review of Scene-Text-Detection and Recognition”, Archives of Computational Methods in Engg, 2019.

L. Deng, Y. Gong, X Lu, Z Ma, M Xie, "STELA: A Real Time Scene-Text-Detector with Learned Anchor", Submitted to cornell University, arXiv:1909.07549, 2019.

M. Liao, B. Shi, W. Liu,” TextBoxes- A Fast Text-Detector with a Single Deep-Neural-Network”, AAAI, 2017.

M. Liao, B. Shi, X. Bai. TextBoxes++, "A Single-Shot Oriented Scene-Text-Detector",. arXiv:1801.02765, Submitted on 27 Apr 2018 to Cornell University 2018.

M. Liao, Z. Wan, C. Yao," Real-time Scene-TextDetection with Differentiable Binarization", Conference on Artificial Intelligence, 2020.

N.Howe, "A Laplacian Energy for Document Binarization", ICDAR, 2011.

P. Adarsh, P. Rathi, " YOLO v3 Tiny- Object Detection and Recognition using one-stage improved model", ICACCS, 2020.

T. Kasar, J. Kumar, "Font and Background Color Independent Text-Binarization”, International Workshop on Camera Based Document Analysis and Recognition, pp. 3-9, 2007.

W. Wang, E. Xie, Y. Zang, T. Lu,” Efficient and Accurate Arbitrary-Shaped Text-Detection with Pixel Aggregation Network”, ICCV, 2019.

X. Rong, C. Yi, Y. Tian ,"Unambiguous text-localization and retrieval for cluttered scenes", In CVPR, 2017, pp. 3279-3287.

X. Zhou., C. Yao, H. Wen, Y. Wang,” East: an efficient and accurate scene-text-detector", arXiv preprint arXiv:1704.03155, Submitted on 10 Jul 2017 to Cornell University.

X Liu, G. Meng, C. Pan, "Scene-text-detection and recognition with advances in deep-learning: a survey", IJDAR 2019.

Z. Huang, Z. Zhong, Q. Huo," Mask RCNN with PyramidAttention-Network for Scene-Text-Detection", IEEE Conf on App of Comp Vis 2019.

Z. Zhang, W. Shen, , C. Yao," Symmetry-based text line detection in natural scenes", In CVPR, 2015, pp. 2558-2567. 